\title{
Ergodic Ramsey theory: a dynamical approach to static theorems
}

\author{
Vitaly Bergelson*
}

\begin{abstract}
We discuss classical results of Ramsey theory together with their dynamical counterparts, survey recent developments and formulate some natural open questions and conjectures.
\end{abstract}

Mathematics Subject Classification (2000). Primary 28D15, 05D10; Secondary 37A45, 37B10.

Keywords. Amenable group, ergodic theory, Furstenberg's correspondence principle, nilmanifold, Ramsey theory, recurrence, Stone-Čech compactification.

\section{Introduction}

Since its inception, ergodic theory was successfully employing combinatorial ideas and methods - from the use of the ubiquitous pigeonhole principle in the proof of the Poincaré recurrence theorem to combinatorial proofs of maximal inequalities, to the utilization of the marriage lemma in Ornstein's isomorphism theory. This debt to combinatorics is amply repaid by the accomplishments of the ergodic Ramsey theory.

Ergodic Ramsey theory was initiated in 1977 when H. Furstenberg [F3] proved a far reaching extension of the classical Poincaré recurrence theorem and derived from it the celebrated Szemerédi's theorem [Sz], which states that every set $E \subset \mathbb{N}$ with $\bar{d}(E):=\lim _{\sup } \rightarrow \infty \frac{|E \cap\{1,2, \ldots, N\}|}{N}>0$ contains arbitrarily long arithmetic progressions. Furstenberg's ergodic approach to Szemerédi's theorem has not only revealed the dynamical underpinnings of this seemingly static result, but has also opened new vistas for mutually perpetuating research in ergodic theory, combinatorics, and number theory.

This survey is organized as follows. In Section 1 we formulate some of the classical theorems of Ramsey theory and discuss their dynamical counterparts. The subsequent sections are devoted to more recent developments and contain formulations of some natural open questions and conjectures. Unfortunately, due to space constraints, some of the topics will not get the attention they deserve. The readers will find more material together with much more elaborated discussion in the recent survey [B4]. (See also [F4], [B1] and [B3].)

*This work was supported in part by NSF grant DMS-0345350.

Proceedings of the International Congress of Mathematicians, Madrid, Spain, 2006

(C) 2006 European Mathematical Society 


\section{A brief survey}

In partition Ramsey theory the focus is on patterns which can always be found in one cell of any finite partition of a highly organized structure such as $\mathbb{Z}^{d}$, a complete graph, a vector space, etc. ${ }^{1}$ Here are some examples.

Theorem 1.1 (Gallai-Grünwald ${ }^{2}$ ). For all $r, d \in \mathbb{N}$, if $\mathbb{Z}^{d}=\bigcup_{i=1}^{r} C_{i}$, then one of $C_{i}$ has the property that for every finite set $B \subset \mathbb{Z}^{d}$, there exist $n \in \mathbb{N}$ and $v \in \mathbb{Z}^{d}$ such that $v+n B=\{v+n u: u \in B\} \subset C_{i}$. In other words, $C_{i}$ contains a homothetic image of every finite set.

Theorem 1.2 (cf. [GraRS], p. 40). Let $V$ be an infinite vector space over a finite field. For all $r \in \mathbb{N}$, if $V=\bigcup_{i=1}^{r} C_{i}$, then one of $C_{i}$ contains affine vector spaces of arbitrary finite dimension.

Theorem 1.3 (Hindman's Theorem, [Hi]). Given an infinite set $E=\left\{x_{1}, x_{2}, \ldots\right\}$ of natural numbers let $F S(E)=\left\{x_{i_{1}}+x_{i_{2}}+\cdots+x_{i_{k}}: i_{1}<i_{2}<\cdots<i_{k}, k \in \mathbb{N}\right\}$. For all $r \in \mathbb{N}$, if $\mathbb{N}=\bigcup_{i=1}^{r} C_{i}$, then one of $C_{i}$ contains a set of the form $F S(E)$ for some infinite set $E \subset \mathbb{N}$.

The density Ramsey theory attempts to explain (and enhance) the results of the partition Ramsey theory by studying the patterns which ought to appear in any "large" set. The notion of largeness may vary but it is always assumed to be partition regular in the sense that for any finite partition of a large set at least one of the cells is large. One also assumes (of course) that the family of large subsets of the ambient structure $S$ includes $S$ itself. We will formulate now density results which correspond to (and refine) the partition results contained in Theorems 1.1, 1.2, and 1.3.

Theorem 1.4 (Furstenberg-Katznelson's multidimensional Szemerédi theorem, [FK1]). Let $d \in \mathbb{N}$ and assume that $E \subset \mathbb{Z}^{d}$ is a set of positive upper density, that is

$$
\bar{d}(E)=\limsup _{N \rightarrow \infty} \frac{\left|E \cap[-N, N]^{d}\right|}{(2 N+1)^{d}}>0 .
$$

For every finite set $B \subset \mathbb{Z}^{d}$ there exist $n \in \mathbb{N}$ and $v \in \mathbb{Z}^{d}$ such that $v+n B \subset E$.

Let $F$ be a finite field and let $V_{F}$ be a countably infinite vector space over $F$. To define a notion of largeness which will allow us to formulate a density version of Theorem 1.2, observe that, as an abelian group, $V_{F}$ is isomorphic to the direct sum $F_{\infty}$ of countably many copies of $F$ :

$$
F_{\infty}=\left\{\left(a_{1}, a_{2}, \ldots\right): a_{i} \in F \text { and all but finitely many } a_{i}=0\right\}=\bigcup_{n=1}^{\infty} F_{n},
$$

\footnotetext{
${ }^{1}$ More precisely, one either deals with arbitrary finite partitions of an infinite structure or with partitions into a fixed number of cells of sufficiently large finite structures.

${ }^{2}$ Grun̈wald (who later changed his name to Gallai) apparently never published his proof. See [R], p. 123 and [GraRS], p. 38.
} 
where $F_{n}=\left\{\left(a_{1}, a_{2}, \ldots\right): a_{i}=0\right.$ for $\left.i>n\right\} \cong F \oplus \cdots \oplus F$ ( $n$ times). For a set $E \subset V_{F}$ (where $V_{F}$ is identified with $F_{\infty}$ ), define the upper density $\bar{d}(E)$ as ${ }^{3}$

$$
\bar{d}(E)=\limsup _{n \rightarrow \infty} \frac{\left|E \cap F_{n}\right|}{\left|F_{n}\right|} .
$$

Theorem 1.5. Every set of positive upper density in the vector space $V_{F}$ contains affine subspaces of every finite dimension.

Before introducing a family of notions of largeness which is pertinent to Hindman's theorem (Theorem 1.3 above), let us remark that the naive attempt to use the notion of upper density (which works well for the Szemerédi-type theorems) immediately fails. Indeed, while the set of odd integers has density $\frac{1}{2}$, it clearly does not contain the sum of any two of its elements. One can actually construct, for any $\varepsilon>0$, a set $S \subset \mathbb{N}$ with $d(S)=\lim _{N \rightarrow \infty} \frac{|S \cap\{1,2, \ldots, N\}|}{N}>1-\varepsilon$, which does not contain $F S(E)$ for any infinite set $E \subset \mathbb{N}$.

It turns out that a natural notion of largeness appropriate for Hindman's theorem can be introduced with the help of $\beta \mathbb{N}$, the Stone-Čech compactification of $\mathbb{N}$. In view of the increasingly important role which Stone-Čech compactifications play in ergodic Ramsey theory, we will briefly discuss some of the relevant definitions and facts. For missing details see ([B1], Section 3) and [B3]. (See also [HiS] for a comprehensive treatment of topological algebra in Stone-Čech compactifications.)

A convenient way of dealing with $\beta \mathbb{N}$ is to view it as an appropriately topologized set of ultrafilters on $\mathbb{N}$. Recall that an ultrafilter $p$ on $\mathbb{N}$ is a maximal filter, namely a family of subsets of $\mathbb{N}$ satisfying the following conditions (the first three of which constitute, for a nonempty family of sets, the definition of a filter).

(i) $\varnothing \notin p$;

(ii) $A \in p$ and $A \subset B$ imply $B \in p$;

(iii) $A \in p$ and $B \in p$ imply $A \cap B \in p$;

(iv) (maximality) if $r \in \mathbb{N}$ and $\mathbb{N}=\bigcup_{i=1}^{r} C_{i}$ then for some $i \in\{1,2, \ldots, r\}$, $C_{i} \in p$.

One can naturally identify each ultrafilter $p$ with a finitely additive $\{0,1\}$-valued probability measure $\mu_{p}$ on the power set $\mathcal{P}(\mathbb{N})$. This measure $\mu_{p}$ is defined by the requirement $\mu_{p}(C)=1$ iff $C \in P$. Without saying so explicitly, we will always think of ultrafilters as such measures, but will prefer to write $C \in p$ instead of $\mu_{p}(C)=1$.

Any $n \in \mathbb{N}$ defines the so-called principal ultrafilter $\{C \subset \mathbb{N}: n \in C\}$. Principal ultrafilters can be viewed as point measures corresponding to elements of $\mathbb{N}$, and are the only ones which can be constructed without the use of Zorn's lemma (see [CN], pp. 161-162). Since ultrafilters are maximal filters, any family of subsets of $\mathbb{N}$ which

\footnotetext{
${ }^{3}$ This definition depends, of course, on the way $V_{F}$ is represented as an infinite direct sum. Each such representation leads to a notion of upper density in $V_{F}$.
} 
has the finite intersection property can be "extended" to an ultrafilter. Given $C \subset \mathbb{N}$, let $\bar{C}=\{p \in \beta \mathbb{N}: C \in p\}$. The family $g=\{\bar{C}: C \in \mathbb{N}\}$ forms a basis for the open sets of a topology on $\beta \mathbb{N}$ (as well as a basis for the closed sets), and, with this topology, $\beta \mathbb{N}$ is a compact Hausdorff space. Clearly, $\overline{\mathbb{N}}=\beta \mathbb{N}$, which hints that the operation of addition (and that of multiplication, as well) can be extended from $\mathbb{N}$ to $\beta \mathbb{N}$. In the following definition, $C-n$ (where $C \subset \mathbb{N}$ and $n \in \mathbb{N}$ ) is the set of all $m$ such that $m+n \in C$. For $p, q \in \beta \mathbb{N}$, define

$$
p+q=\{A \subset \mathbb{N}:\{n \in \mathbb{N}:(A-n) \in p\} \in q\}
$$

It is not hard to check that for principal ultrafilters the operation + corresponds to addition in $\mathbb{N}$. One can show that $p+q$ is an ultrafilter, that the operation + is associative and that, for any fixed $p \in \beta \mathbb{N}$, the function $\lambda_{p}(q)=p+q$ is a continuous self-map of $\beta \mathbb{N}$. It follows that with the operation,$+ \beta \mathbb{N}$ becomes a compact left topological semigroup. By a theorem due to R. Ellis, [E], any such semigroup has an idempotent. It turns out the idempotent ultrafilters in $(\beta \mathbb{N},+)$ (viewed as measures) have a natural shift-invariant property which is responsible for a variety of applications including the following result which may be regarded as a density version of Hindman's theorem.

Theorem 1.6. Let $p$ be an idempotent ultrafilter in $(\beta \mathbb{N},+)$. If $C \in p$, then there is an infinite set $E \subset \mathbb{N}$ such that $F S(E) \subset C$.

While Theorems 1.1 through 1.6 obviously have a common Ramsey-theoretical thread, their formulations do not reveal much about their dynamical content. Our next goal is to convince the reader that all of these results can be interpreted as recurrence theorems in either topological or measure-preserving dynamics. (Topological dynamics forms the natural framework for partition results; measure preserving dynamics corresponds to density statements).

We start with formulating the dynamical version of the Gallai-Grünwald theorem. The idea to apply the methods of topological dynamics to partition results is due to H. Furstenberg and B. Weiss (See $[\mathrm{FW}]$ ).

Theorem 1.7 (cf. [FW], Theorem 1.4). Let $d \in \mathbb{N}, \varepsilon>0$, and let $X$ be a compact metric space. For any finite set of commuting homeomorphisms $T_{i}: X \rightarrow X, i=$ $1,2, \ldots, k$, there exist $x \in X$ and $n \in \mathbb{N}$ such that $\operatorname{diam}\left\{x, T_{1}^{n} x, T_{2}^{n} x, \ldots, T_{k}^{n} x\right\}<\varepsilon$.

To derive Theorem 1.1 from Theorem 1.7, one utilizes the fact that, for fixed $r, d \in$ $\mathbb{N}$, the $r$-colorings of $\mathbb{Z}^{d}$ (viewed as mappings from $\mathbb{Z}^{d}$ to $\{1,2, \ldots, r\}$ ) are naturally identified with the points of the compact product space $\Omega=\{1,2, \ldots, r\}^{\mathbb{Z}^{d}}$. For $m=\left(m_{1}, m_{2}, \ldots, m_{d}\right) \in \mathbb{Z}^{d}$, let $|m|=\max _{1 \leq i \leq d}\left|m_{i}\right|$. Introduce a metric on $\Omega$ by defining, for any pair $x, y \in \Omega, \rho(x, y)=\inf _{n \in \mathbb{N}}\left\{\frac{1}{n}: x(m)=y(m)\right.$ for $\left.|m|<n\right\}$. Note that $\rho(x, y)<1 \Longleftrightarrow x(0)=y(0)$. Let $B=\left\{b_{1}, \ldots, b_{k}\right\} \subset \mathbb{Z}^{d}$. Define the homeomorphisms $T_{i}: \Omega \rightarrow \Omega, i=1,2, \ldots, k$ by $\left(T_{i} x\right)(m)=x\left(m+a_{i}\right)$, and set, for $l=\left(l_{1}, l_{2}, \ldots, l_{k}\right) \in \mathbb{Z}^{k}, T^{l}=T_{1}^{l_{1}} T_{2}^{l_{2}} \cdots T_{k}^{l_{k}}$. Let $y(m), m \in \mathbb{Z}^{d}$, be the element 
of $\Omega$ which corresponds to the given coloring $\mathbb{Z}^{d}=\bigcup_{i=1}^{r} C_{i}$. let $X={\overline{\left\{T^{l} y\right\}}}_{l \in \mathbb{Z}^{k}}$ be the orbital closure of $x$ in $\Omega$. It follows from Theorem 1.7 that for some $x \in X$ which can without loss of generality be chosen to be of the form $T^{u} y$ for some $u \in \mathbb{Z}^{k}$, one has $\operatorname{diam}\left\{x, T_{1}^{n} x, T_{2}^{n} x, \ldots, T_{k}^{n} x\right\}=\operatorname{diam}\left\{T^{u} y, T^{u} T_{1}^{n} y, T^{u} T_{2}^{n} y, \ldots, T^{u} T_{k}^{n} y\right\}<1$. This implies that, for $v=u_{1} b_{1}+u_{2} b_{2}+\cdots+u_{k} b_{k}, y(v)=y\left(v+n b_{1}\right)=\cdots=$ $y\left(v+n b_{k}\right)$, which means that the set $v+n B$ is monochromatic.

In a similar fashion one can derive Theorem 1.2 from the following dynamical result. (Cf. [B4], p. 766).

Theorem 1.8. Let $F$ be a finite field and let $F_{\infty}$ be the direct sum of countably many copies of $F$. Assume that $\left(T_{g}\right)_{g \in F_{\infty}}$ is an action of $F_{\infty}$ by homeomorphisms on a compact metric space $X$. Then for all $\varepsilon>0$, there exist $x \in X$ and $g \in F_{\infty}$, $g \neq(0,0, \ldots)$ such that $\operatorname{diam}\left\{T_{c g} x, c \in F\right\}<\varepsilon$.

We move now to dynamical formulations of Theorems 1.4 and 1.5. We start with the discussion of Szemerédi's theorem (corresponding to $d=1$ in Theorem 1.4). Let $E \subset \mathbb{N}$ with $\bar{d}(E)>0$. Observe that $E$ contains a progression of the form $\{a, a+n, \ldots, a+k n\}$ if and only if $E \cap(E-n) \cap \cdots \cap(E-k n) \neq \emptyset$. It is not too hard to see that Szemerédi's theorem is actually equivalent to an ostensibly stronger statement: for all $k \in \mathbb{N}$ there exists $n \in \mathbb{N}$ such that $\bar{d}(E \cap(E-n) \cap \cdots \cap(E-k n))>0$. This version of Szemerédi's theorem has already a detectible dynamical content (arithmetic progressions are "produced" as the result of shifting $E$ by $n, 2 n, \ldots, k n$ and getting the intersection of positive upper density). This dynamical essence of Szemerédi's theorem is embodied in Furstenberg's multiple recurrence theorem (which implies Szemerédi's result):

Theorem 1.9 ([F3]). Let $T$ be a measure preserving transformation of a probability measure space $(X, \mathcal{B}, \mu)$. For all $k \in \mathbb{N}$ and all $A \in \mathscr{B}$ with $\mu(A)>0$, there exists $n \in \mathbb{N}$ such that

$$
\mu\left(A \cap T^{-n} A \cap T^{-2 n} A \cap \cdots \cap T^{-k n} A\right)>0 .
$$

To derive Szemerédi's theorem from Theorem 1.9 one uses the Furstenberg's correspondence principle which allows one to connect the dynamics in the "pseudodynamical" system $(\mathbb{Z}, \mathcal{P}(\mathbb{Z}), \bar{d}, \tau)$ (where $\tau$ is the shift map: $\tau(n)=n+1, n \in \mathbb{Z}$ ) with a genuine measure preserving symbolic system which can be naturally constructed given a set $E \subset \mathbb{Z}$ with $\bar{d}(E)>0$.

Let $E$ be a set of integers with $\bar{d}(E)>0$ and let $X$ be the orbital closure of $1_{E} \in\{0,1\}^{\mathbb{Z}}$ under the transformation $T: \omega(l) \rightarrow \omega(l+1), \omega \in\{0,1\}^{\mathbb{Z}}$. Let $C=\{\omega \in X: \omega(0)=1\}$. One can show (see, for example, [F4], Lemma 3.17) that there exists a $T$-invariant Borel measure $\mu$ on $X$ which satisfies $\mu(C) \geq \bar{d}(E)$. By Theorem 1.9 there exists $n \in \mathbb{N}$ such that $\mu\left(C \cap T^{-n} C \cap T^{-2 n} C \cap \cdots \cap T^{-k n} C\right)>0$. If $\omega \in C \cap T^{-n} C \cap T^{-2 n} C \cap \cdots \cap T^{-k n} C$ then $\left\{\omega, T^{n} \omega, T^{2 n} \omega, \ldots, T^{k n} \omega\right\} \in C$, which implies $\omega(0)=\omega(n)=\omega(2 n)=\cdots=\omega(k n)$. Since $\omega$ belongs to the orbital closure of $1_{E}$, there is an $m \in \mathbb{Z}$ such that the sequences $\omega(l)$ and $T^{m} 1_{E}(l)$ coincide for 
$0 \leq l \leq k n$. This implies $1_{E}(m)=1_{E}(m+n)=1_{E}(m+2 n)=\cdots=1_{E}(m+k n)$ and gives a progression $\{m, m+n, \ldots, m+k n\} \subset E$.

Similar considerations (involving Furstenberg's correspondence principle for $\mathbb{Z}^{d}$ - and $F_{\infty}$-actions) allow one to derive Theorems 1.4 and 1.5 from the following dynamical theorems.

Theorem 1.10 ([FK1]). Let $(X, \mathcal{B}, \mu)$ be a probability measure space. For any finite set $\left\{T_{1}, T_{2}, \ldots, T_{k}\right\}$ of commuting measure-preserving transformations of $X$ and for all $A \in \mathscr{B}$ with $\mu(A)>0$, there exists $n \in \mathbb{N}$ such that

$$
\mu\left(A \cap T_{1}^{-n} A \cap T_{2}^{-n} A \cap \cdots \cap T_{k}^{-n} A\right)>0 .
$$

Theorem 1.11. Let $\left(T_{g}\right)_{g \in F_{\infty}}$ be a measure preserving action of $V_{F}=F_{\infty}$ on a probability measure space $(X, \mathcal{B}, \mu)$. Let $A \in \mathcal{B}, \mu(A)>0$. Then for some $g \in F_{\infty}, g \neq(0,0, \ldots)$, one has $\mu\left(\bigcap_{c \in F} T_{c g} A\right)>0 .{ }^{4}$

Remark. It is not hard to derive from Theorem 1.11 (by simple iterating procedure) the following fact: for all $k \in \mathbb{N}$ there exist $g_{1}, \ldots, g_{k} \in F_{\infty}$ such that $\operatorname{dim}\left(\operatorname{span}\left\{g_{1}, g_{2}, \ldots, g_{k}\right\}\right)=k$ and $\mu\left(\bigcap_{i=1}^{k} \bigcap_{c \in F} T_{c g_{i}} A\right)>0$. It is this result, which, via the appropriate version of Furstenberg's correspondence principle, implies Theorem 1.5.

We will briefly discuss now the dynamical content of Hindman's theorem. Let $p \in \beta \mathbb{N}$ be an idempotent ultrafilter. The relation $p=p+p$ implies (via (1)) that a set $C \subset \mathbb{N}$ is $p$-large (i.e. belongs to $p$ ) if and only if $\{n \in \mathbb{N}:(C-n) \in p\} \in p$. In other words, if $p \in \beta \mathbb{N}$ is an idempotent, then every $p$-large set has the property that, for $p$-many $n \in \mathbb{N}$, the shifted set $C-n$ is also $p$-large. This, in turn, means that, if $C$ is $p$-large then for $p$-many $n$, the set $C \cap(C-n)$ is $p$-large. This can be interpreted as a version of the Poincare recurrence theorem for idempotent ultrafilter measures. The fact that the "shifting" $n$ can itself be chosen to belong to the $p$-large set $C$ comes as a bonus which, as we will presently see, immediately leads to a short and streamlined proof of Hindman's theorem. ${ }^{5}$ Fix an idempotent ultrafilter $p$ and let $\mathbb{N}=\bigcup_{i=1}^{r} C_{i}$ be an arbitrary finite partition of $\mathbb{N}$. Since $p$ is a finitely additive probability measure, one (and only one) of $C_{i}$, call it $C$, will be $p$-large. As we have seen above, we can choose $n_{1} \in C$ so that the set $C_{1}=C \cap\left(C-n_{1}\right)$ is in $p$. We can now choose $n_{2} \in C_{1}$ so that $n_{2}>n_{1}$ and $C_{2}=C_{1} \cap\left(C_{1}-n_{2}\right)=C \cap\left(C-n_{1}\right) \cap\left(C-n_{2}\right) \cap\left(C-\left(n_{1}+n_{2}\right)\right)$ is in $p$. Continuing in this fashion we will obtain an infinite set $E=\left\{n_{1}, n_{2}, \ldots\right\}$ such that $F S(E) \subset C$, which concludes the proof of Hindman's theorem.

The sets of finite subsets which appear in Hindman's theorem are called in ergodic Ramsey theory IP sets (for infinite-dimensional parallelepiped, a term coined by H. Furstenberg and B. Weiss ${ }^{6}$ ). The notion of IP set makes sense in any commutative

\footnotetext{
${ }^{4}$ Theorem 1.11 follows, for example, from Theorems 1.13 and 2.9 below.

${ }^{5}$ The original proof of Hindman's theorem in [Hi] was very complicated. See [HiS], pp. 102-103 for references to other proofs and interesting historical comments.

${ }^{6}$ As we have seen, IP also naturally connects to IdemPotent.
} 
semigroup and it is often convenient to think of IP sets as generalized semigroups. Many recurrence and convergence theorems which deal with semigroup actions can be extended to actions "along" IP sets, which leads to significant strengthening (and unification) of known results. As an illustration, we will formulate now IP versions of Theorems 1.7 and 1.10 .

An $\mathcal{F}$-sequence in an arbitrary space $Y$ is a sequence $\left(y_{\alpha}\right)_{\alpha \in \mathcal{F}}$ indexed by the set $\mathcal{F}$ of the finite nonempty subsets of $\mathbb{N}$. If $Y$ is a commutative and multiplicative semigroup, one says that an $\mathcal{F}$-sequence defines an IP-system if for any $\alpha=$ $\left\{i_{1}, i_{2}, \ldots, i_{m}\right\} \in \mathcal{F}$ one has $y_{\alpha}=y_{i_{1}} y_{i_{2}} \ldots y_{i_{m}}$. Note that if $\alpha \cap \beta=\emptyset$, then $y_{\alpha \cup \beta}=y_{\alpha} y_{\beta}$. This "partial" semigroup property turns out to be sufficient to guarantee the validity of the following multiple recurrence result which simultaneously extends Theorems 1.7 and 1.8. (It was proved first in [FW]. For a shorter proof based on the idea from [BPT], see [B2] and [B4], Cor. 2.3.)

Theorem 1.12. If $X$ is a compact metric space and $G$ a commutative group of its homeomorphisms, then for any $k$ IP-systems $\left(T_{\alpha}^{(1)}\right)_{\alpha \in \mathcal{F}}, \ldots,\left(T_{\alpha}^{(k)}\right)_{\alpha \in \mathcal{F}}$ in $G$, and all $\varepsilon>0$, there exists $\alpha \in \mathcal{F}$ and $x \in X$ such that $\operatorname{diam}\left\{x, T_{\alpha}^{(1)} x, T_{\alpha}^{(2)} x, \ldots, T_{\alpha}^{(k)} x\right\}<\varepsilon$.

It is clear that Theorem 1.12 implies Theorem 1.7 (any $\mathbb{Z}$ action can be viewed as a special case of an IP-system). But it is also not hard to see that Theorem 1.12 implies Theorem 1.8. See [B4], pp. 765-766 for details.

IP sets can be conveniently utilized to measure the abundance of configurations which are studied in Ramsey theory. Call a set $E$ in a commutative semigroup $G$ an IP* set if $E$ has nonempty intersection with every IP set in $G$. It is not hard to show that every $\mathrm{IP}^{*}$ set is syndetic. ${ }^{7}$ To see this, one has to observe that the complement of a non-syndetic set has to contain arbitrarily long intervals, and it is not hard to show that any such set contains an IP set.

The advantage of IP* sets over syndetic sets is that the family of IP* sets has the finite intersection property (this can be shown with the help of Hindman's theorem). It follows that Theorem 1.12 not only gives a simultaneous extension of Theorems 1.7 and 1.8, but also refines each of them. For example, it follows from Theorem 1.12 that for any finite partition $\mathbb{N}=\bigcup_{i=1}^{r} C_{i}$, one of $C_{i}$ has the property that, for any $k \in \mathbb{N}$, the set

$$
R_{k}=\left\{d \in \mathbb{N}: \text { for some } m \in \mathbb{N},\{m, m+d, m+2 d, \ldots, m+k d\} \subset C_{i}\right\}
$$

is IP*. We will see in the next section that this set $R_{k}$ has much stronger intersectivity properties. (For example, $R_{k}$ has nontrivial intersection with the set of values of any integer-valued polynomial $p(n)$ satisfying $p(0)=0)$.

The following powerful ergodic IP Szemerédi theorem obtained by H. Furstenberg and Y. Katznelson in [FK2] is a natural measure preserving analogue of Theorem 1.12.

\footnotetext{
${ }^{7}$ A subset $S$ in a discrete semigroup $G$ is called syndetic if finitely many translates of $S$ cover $G$. If $G$ is not commutative one has to distinguish between the notions of left and right syndetic. A left translate of $S$ is defined as $x^{-1} S=\{g \in G: x g \in S\}$ and a right translate is defined as $S x^{-1}=\{g \in G: g x \in S\}$.
} 
Theorem 1.13 (See [FK2], Theorem A). Let $(X, \mathcal{B}, \mu)$ be a probability space and $G$ an abelian group of measure-preserving transformations of $X$. For all $k \in \mathbb{N}$, any IP-systems $\left(T_{\alpha}^{(1)}\right)_{\alpha \in \mathcal{F}},\left(T_{\alpha}^{(2)}\right)_{\alpha \in \mathcal{F}}, \ldots,\left(T_{\alpha}^{(k)}\right)_{\alpha \in \mathcal{F}}$ in $G$ and all $A \in \mathcal{B}$ with $\mu(A)>0$ there exists $\alpha \in \mathcal{F}$ such that

$$
\mu\left(A \cap T_{\alpha}^{(1)} A \cap T_{\alpha}^{(2)} A \cap \cdots \cap T_{\alpha}^{(k)} A\right)>0 .
$$

Since the notion of an IP-system of commuting invertible measure preserving transformations generalizes the notion of a measure preserving action of a countable abelian group, Theorems 1.10 and 1.11 are immediate corollaries of Theorem 1.13. It also follows that, on a combinatorial level, Theorem 1.13 implies Theorems 1.4 and 1.5. However, Theorem 1.13 gives more! For example, it follows from it that the sets of configurations always to be found in "large" sets in $\mathbb{Z}^{d}$ or $F_{\infty}$ are abundant in the sense that their parameters form IP* sets. These IP* versions of combinatorial results can be derived, with the help of an appropriate version of Furstenberg's correspondence principle, from the following corollary of Theorem 1.13.

Theorem 1.14. Let $(X, \mathcal{B}, \mu)$ be a probability space, and let $G$ be a countable Abelian group. For all $k \in \mathbb{N}$ and any measure preserving actions $\left(T_{g}^{(1)}\right)_{g \in G}$, $\left(T_{g}^{(2)}\right)_{g \in G}, \ldots,\left(T_{g}^{(k)}\right)_{g \in G}$ of $G$ on $(X, \mathcal{B}, \mu)$ and any $A \in \mathcal{B}$ with $\mu(A)>0$, the set

$$
\left\{g \in G: \mu\left(A \cap T_{g}^{(1)} A \cap T_{g}^{(2)} A \cap \cdots \cap T_{g}^{(k)} A\right)>0\right\}
$$

is an IP* set in $G$ (and in particular, is syndetic).

We will conclude this section by discussing two more classical results of Ramsey theory - the Hales-Jewett partition theorem and its density version proved in [FK3].

Consider the following generalization of tic-tac-toe: $r$ players are taking turns in placing the symbols $s_{1}, \ldots, s_{r}$ in the $k \times k \times \cdots \times k(n$ times) array which one views as the $n^{\text {th }}$ cartesian power $A^{n}$ of a $k$-element set $A=\left\{a_{1}, a_{2}, \ldots, a_{k}\right\}$. (In the classical tic-tac-toe, we have $r=2, k=3, n=2$ ). We are going to define now the notion of a combinatorial line in $A^{n}$. Identify the elements of $A^{n}$ with the set $W_{n}(A)$ of words of length $n$ over the alphabet $A$. Let $\tilde{A}=A \cup\{t\}$ be an extension of the alphabet $A$ obtained by adding a new symbol $t$, and let $W_{n}(t)$ be the set of words of length $n$ over $\tilde{A}$ in which the symbol $t$ occurs. Given a word $w(t) \in W_{n}(t)$ let us define a combinatorial line as a set $\left\{w\left(a_{1}\right), w(a) 2, \ldots, w\left(a_{k}\right)\right\}$ obtained by substituting for $t$ the elements of $A$. For example, the word $43 t 241 t 2$ over the alphabet $\{1,2,3,4,5\} \cup\{t\}$ gives rise to the combinatorial line

$$
\text { \{43124112, 43224122, 43324132, 43424142, 43524152\}. }
$$

It is convenient to think of symbols $s_{1}, \ldots, s_{r}$ as colors; the goal of the players (in our slightly modified tic-tac-toe) is to obtain a monochromatic combinatorial line. The following theorem of Hales and Jewett [HaJ] implies that for fixed $r, k$ and large enough $n$, the first player can always win. 
Theorem 1.15. Let $k, r \in \mathbb{N}$. There exists $c=c(k, r)$ such that if $n \geq c$, then for any $r$-coloring of the set $W_{n}(A)$ of words of length $n$ over the $k$-letter alphabet $A=\left\{a_{1}, a_{2}, \ldots, a_{k}\right\}$, there is a monochromatic combinatorial line.

One of the signs of the fundamental nature of the Hales-Jewett theorem is that one can easily derive from it its multidimensional version. For $m \in \mathbb{N}$, let $t_{1}, t_{2}, \ldots, t_{m}$ be $m$ distinct variables, and let $w\left(t_{1}, t_{2}, \ldots, t_{m}\right)$ be a word of length $n$ over the alphabet $A \cup\left\{t_{1}, t_{2}, \ldots, t_{m}\right\}$. (We assume that the letters $t_{i}$ do not belong to $A$ ). If $w\left(t_{1}, t_{2}, \ldots, t_{m}\right)$ is a word of length $n$ over $A \cup\left\{t_{1}, t_{2}, \ldots, t_{m}\right\}$ in which all of the variables $t_{1}, t_{2}, \ldots, t_{m}$ occur, the result of the substitution

$\left\{w\left(t_{1}, t_{2}, \ldots, t_{m}\right)\right\}_{\left(t_{1}, t_{2}, \ldots, t_{m}\right) \in A^{m}}=\left\{w\left(a_{i_{1}}, a_{i_{2}}, \ldots, a_{i_{m}}\right): a_{i_{j}} \in A, j=1,2, \ldots, m\right\}$

is called a combinatorial $m$-space. Observe now that if we replace the original alphabet $A$ by $A^{m}$, then a combinatorial line in $W_{n}\left(A^{m}\right)$ can be interpreted as a combinatorial $m$-space in $W_{n m}(A)$. Thus we have the following ostensibly stronger theorem as a corollary of Theorem 1.15.

Theorem 1.16. Let $r, k, m \in \mathbb{N}$. There exists $c=c(r, k, m)$ such that if $n \geq c$, then for any $r$-coloring of the set $W_{n}(A)$ of words of length $n$ over the k-letter alphabet $A$, there exists a monochromatic combinatorial $m$-space.

The Hales-Jewett theorem is truly one of the cornerstones of Ramsey theory. Not only many familiar partition results such as Theorems 1.1 and 1.2 are immediate corollaries of the Hales-Jewett theorem, ${ }^{8}$ but this result is the natural basis of many further generalizations, some of which we will encounter in the next sections. Also, the Hales-Jewett theorem and its various generalizations are utilized in proofs of various multiple recurrence results in measure preserving dynamics. For example, the Hales-Jewett theorem is used in [FK2] in the proof of Theorem 1.13. While the Hales-Jewett theorem was originally proved in a purely combinatorial way, it can also be proved with the help of Stone-Čech compactifications and by using the tools of topological dynamics. Each of these additional proofs leads in its turn to further useful results and ramifications. See [BL2], [BL3], [BBHi], [C].

In anticipation of the discussion in the next section, we are going to formulate two more versions of the Hales-Jewett theorem. Let $\mathcal{F}_{0}$ denote the set of finite (potentially empty) subsets of $\mathbb{N}$.

Given $k \in \mathbb{N}$, write $\mathcal{F}_{0}^{k}$ for the set of $k$-tuples of sets form $\mathcal{F}_{0}$. Let us call any $(k+1)$ element subset of $\mathcal{F}_{0}^{k}$ of the form

$$
\begin{aligned}
& \left\{\left(\alpha_{1}, \alpha_{2}, \ldots, \alpha_{k}\right),\left(\alpha_{1} \cup \gamma, \alpha_{2}, \ldots, \alpha_{k}\right),\right. \\
& \left.\quad\left(\alpha_{1}, \alpha_{2} \cup \gamma, \ldots, \alpha_{k}\right), \ldots,\left(\alpha_{1}, \alpha_{2}, \ldots, \alpha_{k} \cup \gamma\right)\right\}
\end{aligned}
$$

a simplex.

\footnotetext{
${ }^{8}$ To see, for example, that Theorem 1.1 follows from the Hales-Jewett theorem, take $A$ to be a finite field $F$. Then $W_{n}(F)=F^{n}$ has the natural structure of an $n$-dimensional vector space over $F$. It is easy to see that, in this case, a combinatorial $m$-space is an affine $m$-dimensional subspace of $F^{n}$.
} 
Theorem 1.17. For any $k \in \mathbb{N}$ and any finite coloring (= partition) of $\mathcal{F}_{0}^{k}$, there exists a monochromatic simplex.

Here is a dynamical counterpart of Theorem 1.17.

Theorem 1.18. Let $(X, \rho)$ be a compact metric space. For $k \in \mathbb{N}$, let $T_{\left(\alpha_{1}, \alpha_{2}, \ldots, \alpha_{k}\right)}$, $\left(\alpha_{1}, \alpha_{2}, \ldots, \alpha_{k}\right) \in \mathcal{F}_{0}^{k}$ be a family of continuous self-maps of $X$ such that for any $\left(\alpha_{1}, \alpha_{2}, \ldots, \alpha_{k}\right),\left(\beta_{1}, \beta_{2}, \ldots, \beta_{k}\right) \in \mathcal{F}_{0}^{k}$ satisfying $\alpha_{i} \cap \beta_{i}=\emptyset, i=1,2, \ldots, k$, one has

$$
T_{\left(\alpha_{1} \cup \beta_{1}, \alpha_{2} \cup \beta_{2}, \ldots, \alpha_{k} \cup \beta_{k}\right)}=T_{\left(\alpha_{1}, \alpha_{2}, \ldots, \alpha_{k}\right)} T_{\left(\beta_{1}, \beta_{2}, \ldots, \beta_{k}\right)} .
$$

Then for all $\varepsilon>0$ and for all $x \in X$ there exist a nonempty finite set $\gamma$ and a $k$-tuple $\left(\alpha_{1}, \alpha_{2}, \ldots, \alpha_{k}\right) \in \mathscr{F}_{0}^{k}$ such that $\alpha_{i} \cap \gamma=\emptyset, i=1,2, \ldots, k$ and

$\operatorname{diam}\left\{T_{\left(\alpha_{1}, \alpha_{2}, \ldots, \alpha_{k}\right)} x, T_{\left(\alpha_{1} \cup \gamma, \alpha_{2}, \ldots, \alpha_{k}\right)} x, T_{\left(\alpha_{1}, \alpha_{2} \cup \gamma, \ldots, \alpha_{k}\right)} x, \ldots, T_{\left(\alpha_{1}, \alpha_{2}, \ldots, \alpha_{k} \cup \gamma\right)} x\right\}<\varepsilon$.

See [B1] for more discussion of various equivalent forms of the Hales-Jewett theorem and ([BL2], Proposition L) for a proof via topological dynamics.

\section{Ramsey theory and multiple recurrence along polynomials}

We start this section with the formulation of the Furstenberg-Sárközy theorem which has interesting links with spectral theory, Diophantine approximations, combinatorics, and dynamical systems.

Theorem 2.1 ([F4], [Sa]). Let $E \subset \mathbb{N}$ be a set of positive upper density, and let $p(n) \in \mathbb{Z}[n]$ be a polynomial with $p(0)=0$. Then there exist $x, y \in E$ and $n \in \mathbb{N}$ such that $x-y=p(n)$.

This result is quite surprising. While it is not hard to show that the set of differences $E-E=\{x-y: x, y \in E\}$ of a set $E$ with $\bar{d}(E)>0$ is syndetic, there is, a priori, no obvious reason for the set $E-E$ to be so "well spread" as to nontrivially intersect the set of values of every polynomial $p(n) \in \mathbb{Z}[n]$ which vanishes at zero. ${ }^{9}$ The following dynamical counterpart of Theorem 2.1, from which Theorem 2.1 follows with the help of Furstenberg's correspondence principle, is just as striking.

Theorem 2.2. For any invertible measure preserving system $(X, \mathscr{B}, \mu, T)$, any $A \in$ $\mathscr{B}$ with $\mu(A)>0$, and any polynomial $p(n) \in \mathbb{Z}[n]$ with $p(0)=0$, there exists $n \in \mathbb{N}$ such that $\mu\left(A \cap T^{p(n)} A\right)>0$.

The following result obtained in [BL1] gives a simultaneous generalization of Theorem 2.1 and of the Furstenberg-Katznelson multidimensional Szemerédi theorem, Theorem 1.4.

\footnotetext{
${ }^{9}$ T. Kamae and M. Mendès-France have shown in $[\mathrm{KM}]$ that a polynomial $p(n) \in \mathbb{Z}[n]$ has this "intersectivity" property if and only if $\{p(n): n \in \mathbb{Z}\} \cap a \mathbb{Z} \neq \emptyset$ for all $a \in \mathbb{N}$. For example, the polynomial $p(n)=$ $\left(n^{2}-13\right)\left(n^{2}-17\right)\left(n^{2}-221\right)$ is such.
} 
Theorem 2.3 (cf. [BL1], Theorem $\mathrm{B}^{\prime}$ ). Let $r, l \in \mathbb{N}$ and let $P: \mathbb{Z}^{r} \rightarrow \mathbb{Z}^{l}$ be a polynomial mapping satisfying $P(0)=0$. For all $S \subset \mathbb{Z}^{l}$ with $\bar{d}(S)>0$ and for all finite sets $F \subset \mathbb{Z}^{r}$, there exists elements $n \in \mathbb{N}$ and $n \in \mathbb{Z}^{l}$ such that $u+P(n F)=$ $\left\{u+P\left(n x_{1}, n x_{2}, \ldots, n x_{r}\right):\left(x_{1}, x_{2}, \ldots, x_{r}\right) \in F\right\} \subset S$.

The ergodic theoretic result from which Theorem 2.3 is derived in [BL1] involves products of commuting measure preserving transformations evaluated at "polynomial times":

Theorem 2.4 ([BL1], Theorem A). Let, for some $t, k \in \mathbb{N}, p_{1,1}(n), \ldots, p_{1, t}(n)$, $p_{2,1}(n), \ldots, p_{2, t}(n), \ldots, p_{k, 1}(n), \ldots, p_{k, t}(n)$ be polynomials with rational coefficients taking integer values on the integers and satisfying $p_{i, j}(0)=0, i=1,2, \ldots, k$, $j=1,2, \ldots, t$. Then for all probability space $(X, \mathscr{B}, \mu)$, all commuting invertible measure preserving transformations $T_{1}, T_{2}, \ldots, T_{t}$ of $X$ and all $A \in \mathscr{B}$ with $\mu(A)>0$, one has

$$
\liminf _{N \rightarrow \infty} \frac{1}{N} \sum_{n=0}^{n-1} \mu\left(A \cap \prod_{j=1}^{t} T_{j}^{-p_{1, j}(n)} A \cap \prod_{j=1}^{t} T_{j}^{-p_{2, j}(n)} A \cap \cdots \cap \prod_{j=1}^{t} T_{j}^{-p_{k, j}(n)} A\right)>0 .
$$

As we have seen in the previous section, the "linear" multiple recurrence results admit far reaching IP refinements. This leads to the question whether similar IP extensions may be obtained for polynomial results as well. For example, one would like to know whether, for every invertible measure preserving system $(X, \mathcal{B}, \mu, T)$, every polynomial $p(n) \in \mathbb{Z}[n]$ with $p(0)=0$, and every $A \in \mathscr{B}$ with $\mu(A)>0$, the set $\left\{n \in \mathbb{Z}: \mu\left(A \cap T^{p(n)} A\right)>0\right\}$ is an IP* set. In other words, one would like to know whether for every infinite set $\left\{n_{1}, n_{2}, \ldots\right\} \subset \mathbb{N}$ there exists $\alpha \in \mathcal{F}$ such that $\mu\left(A \cap T^{p\left(n_{\alpha}\right)} A\right)>0$, where, as in Section 1, the IP set $\left(n_{\alpha}\right)_{\alpha \in \mathscr{F}}$ is defined by $n_{\alpha}=\sum_{i \in \alpha}, \alpha \in \mathcal{F}$. The answer turns out to be yes and can be obtained by considering, instead of the conventional ergodic averages $\frac{1}{N} \sum_{n=1}^{N} \mu\left(A \cap T^{p(n)} A\right)$, the limits along IP sets (or, alternatively, limits along idempotent ultrafilters). However, a much more important novelty which is encountered when one deals with IP analogues of polynomial recurrence theorems is that one has now a bigger family of functions, namely the IP polynomials, for which the IP versions of familiar theorems make sense.

Let $q\left(t_{1}, \ldots, t_{k}\right) \in \mathbb{Z}\left[t_{1}, \ldots, t_{k}\right]$ and let $\left(n_{\alpha}^{(i)}\right)_{\alpha \in \mathcal{F}}, i=1,2 \ldots, k$, be IP sets in $\mathbb{Z}$. Then $q(\alpha)=q\left(n_{\alpha}^{(1)}, n_{\alpha}^{(2)}, \ldots, n_{\alpha}^{(k)}\right)$ is an example of an IP polynomial. For example, if $\operatorname{deg} q\left(t_{1} \ldots, t_{k}\right)=2$, the $q(\alpha)$ will typically look like

$$
q(\alpha)=\sum_{i=1}^{s} n_{\alpha}^{(i)} m_{\alpha}^{(i)}+\sum_{i=1}^{r} k_{\alpha}^{(i)}
$$

The following result obtained in [BFM] gives an IP extension of Theorem 2.2 for the case of several commuting transformations. 
Theorem 2.5 (cf. [BFM], Corollary 2.1). Suppose that $(X, \mathcal{B}, \mu)$ is a probability space and that $\left\{T_{1}, T_{2}, \ldots, T_{t}\right\}$ is a collection of commuting invertible measure preserving transformations of $X$. Suppose that $\left(n_{\alpha}^{(i)}\right)_{\alpha \in \mathcal{F}} \subset \mathbb{N}$ are IP sets, $i=1,2, \ldots, k$, and that $p_{j}\left(x_{1}, \ldots, x_{k}\right) \in \mathbb{Z}\left[x_{1}, \ldots, x_{k}\right]$ satisfy $p_{j}(0,0, \ldots, 0)=0$ for $j=1,2, \ldots, t$. Then for all $A \in \mathscr{B}$, and all $\varepsilon>0$, there exist $\alpha \in \mathcal{F}$ such that

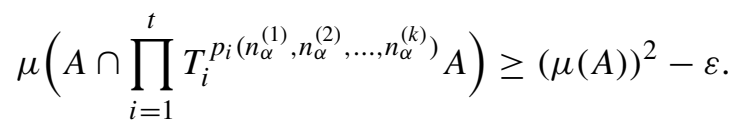

The next natural step is to try to extend Theorem 2.3 to a multiple recurrence result. The following IP polynomial Szemerédi theorem, obtained in [BM2] is an IP extension of Theorem 2.3.

Theorem 2.6 ([BM2], Theorem 0.9). Suppose we are given $t$ commuting invertible measure preserving transformations $T_{1}, \ldots, T_{t}$ of a probability space $(X, \mathscr{B}, \mu)$. Let $k, r \in \mathbb{N}$ and suppose that $p_{i, j}\left(n_{1}, \ldots, n_{k}\right) \in \mathbb{Q}\left[n_{1}, \ldots, n_{k}\right]$ satisfy $p_{i, j}\left(\mathbb{Z}^{k}\right) \subset \mathbb{Z}$ and $p_{i, j}(0,0, \ldots, 0)=0$ for $1 \leq i \leq t, 1 \leq j \leq r$. Then for every $A \in \mathscr{B}$ with $\mu(A)>0$, the set

$$
\left\{\left(n_{1}, \ldots, n_{k}\right) \in \mathbb{Z}^{k}: \mu\left(\bigcap_{j=1}^{r}\left(\prod_{i=1}^{t} T_{i}^{p_{i, j}\left(n_{1}, \ldots, n_{k}\right)} A\right)\right)>0\right\}
$$

is an $I P^{*} \operatorname{set}$ in $\mathbb{Z}^{k}$.

The following corollary of Theorem 2.6 can be viewed as an IP refinement of Theorem 2.5.

Theorem 2.7. Assume that $P: \mathbb{Z}^{r} \rightarrow \mathbb{Z}^{l}, r, l \in \mathbb{N}$ is a polynomial mapping satisfying $P(0)=0$ and let $F \subset \mathbb{Z}^{r}$ be a finite set. Then for all $E \subset \mathbb{Z}^{l}$ with $\bar{d}(E)>0$ and all

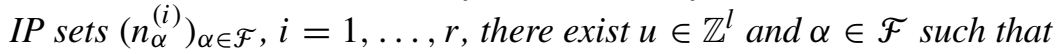

$$
\left\{u+P\left(n_{\alpha}^{(1)} x_{1}, n_{\alpha}^{(2)} x_{2}, \ldots, n_{\alpha}^{(r)} x_{r}\right):\left(x_{1}, \ldots, x_{r}\right) \in F\right\} \subset S .
$$

We would like to mention the two combinatorial facts which play a decisive role in the proof of Theorem 2.6. The first is the Milliken-Taylor theorem ([M], [T]) which was also utilized in the proof of Theorem 2.5. The second is the polynomial Hales-Jewett theorem obtained via topological dynamics in [BL2]. The following formulation of the polynomial Hales-Jewett theorem should be juxtaposed with the formulation of its "linear" case given in Theorem 1.17.

Theorem 2.8. For $k, d \in \mathbb{N}$, let $\mathcal{F}_{0}^{k}\left(\mathbb{N}^{d}\right)$ denote the set of $k$-tuples of finite (possibly empty) subsets of $\mathbb{N}^{d}=\mathbb{N} \times \cdots \times \mathbb{N}$ (d times). For every finite coloring of $\mathcal{F}_{0}^{k}\left(\mathbb{N}^{d}\right)$ there exists a monochromatic simplex of the form

$$
\begin{aligned}
& \left\{\left(\alpha_{1}, \alpha_{2}, \ldots, \alpha_{k}\right),\left(\alpha_{1} \cup \gamma^{d}, \alpha_{2}, \ldots, \alpha_{k}\right),\right. \\
& \left.\quad\left(\alpha_{1}, \alpha_{2} \cup \gamma^{d}, \ldots, \alpha_{k}\right), \ldots,\left(\alpha_{1}, \alpha_{2}, \ldots, \alpha_{k} \cup \gamma^{d}\right)\right\},
\end{aligned}
$$

where $\gamma$ is a finite nonempty subset of $\mathbb{N}$ and $\alpha_{i} \cap \gamma^{d}=\emptyset$ for all $i=1,2, \ldots, k$. 
The polynomial Hales-Jewett theorem plays also a crucial role in the proof of the following recent result of a polynomial nature obtained in [BLM].

Theorem 2.9. Let $V, W$ be finite dimensional vector spaces over a countable field, let $T$ be a measure preserving action of $W$ on a probability measure space $(X, \mathscr{B}, \mu)$ and let $p_{1}, \ldots, p_{k}$ be polynomial mappings $V \rightarrow W$ with zero constant term. Then for all $A \in \mathscr{B}$ with $\mu(A)>0$ there exists $c>0$ such that the set

$$
\left\{v \in V: \mu\left(\bigcap_{i=1}^{k} T_{\left(p_{i}(v)\right)} A\right)>c\right\}
$$

is syndetic in $V$.

Corollary 2.10. Let $p_{1}, \ldots, p_{k}$ be polynomials with integer coefficients and zero constant term. For all $\varepsilon>0$, there exists $N \in \mathbb{N}$ such that whenever $F$ is a field with $|F| \geq N$ and $E \subset F$ with $\frac{|E|}{|F|} \geq \varepsilon$, there exist $v \in F, v \neq 0$, and $w \in E$ such that $p_{i}(v) \neq 0, i=1, \ldots, k$, and $\left\{w, w+p_{1}(v), \ldots, w+p_{k}(v)\right\} \subset E$ for all $i=1, \ldots, k$.

A dynamical counterpart of Theorem 2.8 can be formulated in direct analogy to Theorem 1.8 which corresponds to the "linear" case. (See [B1], Section 4 and [BL2] for more discussion on various equivalent forms of the polynomial Hales-Jewett theorem). Rather than dealing here with the full-fledged dynamical version, we are going to formulate a rather general corollary of the polynomial Hales-Jewett theorem which is suggestive of a further, nilpotent, generalization to be discussed in the next section.

A mapping $\mathcal{P}$ from $\mathcal{F}_{0}$ into a commutative (semi)group $G$ is an IP polynomial of degree 0 if $\mathcal{P}$ is constant, and, inductively, is an IP polynomial of degree $\leq d$ if for all $\beta \in \mathscr{F}_{0}$ there exists an IP polynomial $\mathscr{D}_{\beta} \mathcal{P}: \mathscr{F}_{0}(\mathbb{N} \backslash \beta) \rightarrow G$ of degree $\leq d-1$ (where $\widetilde{F}_{0}(\mathbb{N} \backslash \beta)$ is the set of finite subsets of $\left.\mathbb{N} \backslash \beta\right)$ such that $\mathcal{P}(\alpha \cup \beta)=\mathcal{P}(\alpha)+\left(\mathscr{D}_{\beta} \mathcal{P}\right)(\alpha)$ for every $\alpha \in \mathcal{F}_{0}$ with $\alpha \cap \beta=\emptyset$. (One can easily check that the IP-systems introduced in Section 1 correspond to IP polynomials of degree 1 satisfying $\mathcal{P}(\emptyset)=1_{G}$.)

Theorem 2.11 ([BL2]). Let $G$ be an abelian group of self-homeomorphisms of a compact metric space $(X, \rho)$, let $k \in \mathbb{N}$ and let $\mathcal{P}_{1}, \ldots, \mathcal{P}_{k}$ be IP-polynomials mapping $\mathcal{F}_{0}$ into $G$ and satisfying $\mathcal{P}_{1}(\emptyset)=\cdots=\mathscr{P}_{k}(\emptyset)=1_{G}$. For all $\varepsilon>0$ there exist $x \in X$ and a nonempty $\alpha \in \mathscr{F}_{0}$ such that $\rho\left(\mathscr{P}_{i}(\alpha) x, x\right)<\varepsilon$ for $i=1, \ldots, k$.

We conclude this section by formulating a conjecture about a density version of the polynomial Hales-Jewett theorem which would extend the partition results from [BL2], the Furstenberg-Katznelson density version of the "linear" Hales-Jewett theorem, as well as Theorems 2.4 and 2.9. For $q, d, N \in \mathbb{N}$, let $M_{q, d, N}$ be the set of $q$-tuples of subsets of $\{1,2, \ldots, N\}^{d}$ :

$$
M_{q, d, N}=\left\{\left(\alpha_{1}, \ldots, \alpha_{q}\right): \alpha_{i} \subset\{1,2, \ldots, N\}^{d}, i=1,2, \ldots, q\right\} .
$$


Conjecture 2.12. For all $q, d \in \mathbb{N}$ and $\varepsilon>0$ there exists $c=c(q, d, \varepsilon)$ such that if $N>c$ and a set $S \subset M_{q, d, N}$ satisfies $\frac{|S|}{\left|M_{q, d, N}\right|}>\varepsilon$, then $S$ contains a "simplex" of the form

$$
\begin{aligned}
& \left\{\left(\alpha_{1}, \alpha_{2}, \ldots, \alpha_{q}\right),\left(\alpha_{1} \cup \gamma^{d}, \alpha_{2}, \ldots, \alpha_{q}\right),\right. \\
& \quad\left(\alpha_{1}, \alpha_{2} \cup \gamma^{d}, \ldots, \alpha_{q}\right), \ldots,\left(\alpha_{1}, \alpha_{2}, \ldots, \alpha_{q} \cup \gamma^{d}\right),
\end{aligned}
$$

where $\gamma \subset \mathbb{N}$ is a nonempty set and $\alpha_{i} \cap \gamma^{d}=\emptyset$ for all $i=1, \ldots, q$.

\section{Ergodic Ramsey theory in a noncommutative setting}

The Ramsey theoretical results surveyed in the previous sections deal with commutative (semi)groups. One may wonder whether these results extend to noncommutative structures. A similar question suggests itself with respect to dynamics: is it true that multiple recurrence results such as, say, Theorems 1.10 and 2.4 hold true if the involved transformations do not necessarily commute? It turns out that many of the partition and density theorems (as well as their dynamical counterparts) that we encountered above do hold for nilpotent groups. On the other hand, the analogous results fail quite dramatically for solvable groups of exponential growth. (See Theorem 3.7 below).

We now formulate a nilpotent version of the polynomial Hales-Jewett theorem (see Theorems 2.8 and 2.11), from which one can derive nilpotent extensions of various abelian theorems. In order to do so we have to extend first the notion of a polynomial mapping $\mathcal{P}: \mathscr{F}_{0} \rightarrow G$ (discussed at the end of the previous section) to a nilpotent setup.

If $G$ is an abelian group, one can show (see [BL2], Theorem 8.3) that a mapping $\mathcal{P}: \mathcal{F}_{0} \rightarrow G$ is an IP polynomial of degree $\leq d$ with $P(\emptyset)=1_{G}$ if and only if there exists a family $\left\{g_{j_{1}, \ldots, j_{d}}\right\}_{\left(j_{1}, \ldots, j_{d}\right) \in \mathbb{N}^{d}}$ of elements of $G$ such that for all $\alpha \in \mathcal{F}_{0}$ one has $\mathcal{P}(\alpha)=\prod_{\left(j_{1}, \ldots, j_{d}\right) \in \alpha^{d}} g_{j_{1}, \ldots, j_{d}}$. This characterization of IP polynomials makes sense in the nilpotent setup as well. Given a nilpotent group $G$, let us call a mapping $\mathcal{P}: \mathcal{F}_{0} \rightarrow G$ an IP polynomial if for some $d \in \mathbb{N}$ there exists a family $\left\{g_{j_{1}, \ldots, j_{d}}\right\}_{\left(j_{1}, \ldots, j_{d}\right) \in \mathbb{N}^{d}}$ of elements of $G$ and a linear order $<$ on $\mathbb{N}^{d}$ such that, for any $\alpha \in \mathcal{F}_{0}$, one has $\mathcal{F} P(\alpha)=\prod_{\left(j_{1}, \ldots, j_{d}\right) \in \alpha^{d}}^{<} g_{j_{1}, \ldots, j_{d}}$ (the entries in the product $\prod^{<}$are multiplied in accordance with the order $<$ ). The following nilpotent version of the polynomial Hales-Jewett theorem which was obtained in [BL3] contains many of the above partition results as special cases.

Theorem 3.1 ([BL3], Theorem 0.24). Let $G$ be a nilpotent group of homeomorphisms of a compact metric space $(X, \rho)$ and let $\mathcal{P}_{1}, \ldots, \mathcal{P}_{k}: \mathcal{F}_{0} \rightarrow G$ be IP polynomials satisfying $\mathcal{P}_{1}(\emptyset)=\cdots=\mathcal{P}_{k}(\emptyset)=1_{G}$. Then, for all $\varepsilon>0$, there exist $x \in X$ and $a$ nonempty $\alpha \in \mathcal{F}_{0}$ such that $\rho\left(\mathscr{P}_{i}(\alpha) x, x\right)<\varepsilon$ for all $i=1,2, \ldots, k$. 
One of the corollaries of Theorem 3.1 is the following nilpotent version of Theorem 1.12.

Theorem 3.2 ([BL3], Theorem 0.13). Let $G$ be a nilpotent group of homeomorphisms of a compact metric space $(X, \rho)$, let $k \in \mathbb{N}$ and let $g_{j}^{(i)} \in G, i=1, \ldots, k, j \in \mathbb{N}$. For all $\varepsilon>0$, there exist an element $x$ in $X$ and a nonempty finite subset $\alpha$ of $\mathbb{N}$ such that $\left.\rho\left(\prod_{j \in \alpha} g_{j}^{(i)} x, x\right)\right)<\varepsilon$ for all $i=1, \ldots, k$.

Theorem 3.1 implies also the following results, both of which can be viewed as nilpotent extensions of Theorem 1.2.

Theorem 3.3 (cf. [BL3], Theorem 0.16). Let $q \in \mathbb{N}$ and let $G$ be the multiplicative group of $q \times q$ upper triangular matrices with unit diagonal over an infinite field of finite characteristic. For any finite coloring of $G$ and any $c \in \mathbb{N}$ there exists a subgroup $H$ of $G$ of nilpotency class $q$ and of cardinality $\geq c$, such that for some $h \in G$ the coset $h H$ is monochromatic.

Theorem 3.4 ([BL3], Theorem 0.17). Let $q \in \mathbb{N}$ and $p$ be a prime, with $p>q$. Let $G$ be an infinite free $q$-step nilpotent group with torsion $p$. For any finite coloring of $G$ and any $c \in \mathbb{N}$ there exists a free q-step nilpotent subgroup $H \subset G$ of cardinality $|H| \geq c$, such that, for some $h \in G$, the coset $h H$ is monochromatic.

The following theorem obtained by A. Leibman in [L1] is a nilpotent extension of Theorem 2.4, from which one can also derive a nilpotent generalization of Theorem 2.3.

Theorem 3.5 (cf. [L1], Theorem NM). Let $k, t, r \in \mathbb{N}$. Assume $G$ is a nilpotent group of measure preserving transformations of a probability measure space $(X, \mathcal{B}, \mu)$. Let $p_{i j}\left(n_{1}, \ldots, n_{k}\right) \in \mathbb{Z}\left[n_{1}, \ldots, n_{k}\right]$ with $p_{i j}\left(\mathbb{Z}^{k}\right) \subset \mathbb{Z}$ and $p_{i j}(0,0, \ldots, 0)=0,1 \leq$ $i \leq t, 1 \leq j \leq r$. Then for every $A \in \mathscr{B}$ with $\mu(A)>0$ and any $T_{1}, T_{2}, \ldots, T_{t} \in G$, the set

$$
\left\{\left(n_{1}, \ldots, n_{k}\right) \in \mathbb{Z}^{k}: \mu\left(\bigcap_{j=1}^{r}\left(\prod_{i=1}^{t} T_{i}^{p_{i j}\left(n_{1}, \ldots, n_{k}\right)} A\right)\right)>0\right\}
$$

is syndetic.

There is every reason to believe that nilpotent versions of Theorems 2.6 and 2.9 also hold. The following conjecture, if true, will contain these and many other nilpotent results as special cases.

Conjecture 3.6. Let $G$ be a nilpotent group of measure preserving transformations of a probability measure space $(X, \mathcal{B}, \mu)$, and let $\mathcal{P}_{1}, \ldots, \mathcal{P}_{k}: \mathcal{F}_{0} \rightarrow G$ be IP polynomials. Then for all $A \in \mathscr{B}$ with $\mu(A)>0$ there exists a nonempty $\alpha \subset \mathbb{N}$ such that $\mu\left(A \cap \mathcal{P}_{1}(\alpha) A \cap \cdots \cap \mathcal{P}_{k}(\alpha) A\right)>0$.

Theorem 3.5 raises question whether the assumptions can be further relaxed and whether, in particular, an analogue of Theorem 3.5 holds true if the measure preserving transformations $T_{1}, T_{2}, \ldots, T_{k}$ generate a solvable group. Note that every finitely 
generated solvable group is either of exponential growth or is virtually nilpotent, i.e. it contains a nilpotent group of finite index. (See, for example, [Ro]). Since Theorem 3.5 easily extends to virtually nilpotent groups, the question boils down to solvable groups of exponential growth. The following result answers this question in the negative, in a strong way.

Theorem 3.7 ([BL4], Theorem 1.1 (A)). Assume that $G$ is a finitely generated solvable group of exponential growth. There exists a measure preserving action $\left(T_{g}\right)_{g \in G}$ of $G$ on a probability measure space $(X, \mathcal{B}, \mu)$, elements $g, h \in G$, and a set $A \in \mathscr{B}$ with $\mu(A)>0)$ such that $T_{g^{n}} A \cap T_{h^{n}} A=\emptyset$ for all $n \neq 0$.

It is of interest to know to which extent the property of growth of the acting group alone is responsible for the validity of the positive and negative results formulated above. It was R. Grigorchuk who constructed in [Gri] a large family of groups of intermediate growth, which occupy an intermediate place between the groups of polynomial and exponential growth.

Question 3.8. Which of the above results extend to Grigorchuck's groups?

\section{Generalized polynomials and dynamical systems on nilmanifolds}

As we have seen in the previous section, the nilpotent framework is a natural (and often, ultimate) setup for multiple recurrence and combinatorial applications thereof. It also turns out that dynamical systems on nilmanifolds ${ }^{10}$ are indispensable in solving problems which, on the face of it, have purely abelian character. For example, it is shown in the work of Host and Kra ([HK1]) and Ziegler ([Z]) that one can reduce the problem of establishing the existence of the $L^{2}$ limit

$$
\lim _{N \rightarrow \infty} \frac{1}{N} \sum_{n=0}^{N-1} f_{1}\left(T^{n} x\right) f_{2}\left(T^{2 n} x\right) \ldots f_{k}\left(T^{k n} x\right),
$$

where $T$ is an invertible measure preserving transformation of a probability space $(X, \mathscr{B}, \mu)$ and $f_{i} \in L^{\infty}(X)$, to the study of the special case where $(X, T)$ is a nilsystem. It also turns out that polynomial sequences of nilrotations (see [L2], [L3], [L5]) form an adequate setup for extending Host-Kra's and Ziegler's results to polynomial situations, that is to establishing the existence of the $L^{2}$-limit

$$
\lim _{N \rightarrow \infty} \frac{1}{N} \sum_{n=0}^{N-1} f_{1}\left(T^{p_{1}(n)} x\right) \ldots f_{k}\left(T^{p_{k}(n)} x\right),
$$

\footnotetext{
${ }^{10} \mathrm{~A}$ nilmanifold is a compact homogeneous space $X$ of a nilpotent Lie group $G$; a nilrotation is a translation of $X$ by an element $g \in G, x \mapsto g x$; a nilsystem is a pair $(X, T)$ where $X$ is a nilmanifold and $T$ is a nilrotation on $X$.
} 
where the $p_{i}$ are integer-valued polynomials. See [HK2] and [L4].

Another example, pertaining to recurrence, is given by the following result from [BHKR], the proof of which crucially uses the facts about nilsystems.

Theorem 4.1 ([BHKR]). For every invertible ergodic probability measure preserving system $(X, \mathscr{B}, \mu, T)$, all $A \in \mathscr{B}$ and all $\varepsilon>0$, the sets

$$
\left\{n: \mu\left(A \cap T^{n} A \cap T^{2 n} A\right) \geq \mu(A)^{3}-\varepsilon\right\}
$$

and

$$
\left\{n: \mu\left(A \cap T^{n} A \cap T^{2 n} A \cap T^{3 n} A\right) \geq \mu(A)^{4}-\varepsilon\right\}
$$

are syndetic.

On the other hand, there exists an ergodic system $(X, \mathscr{B}, \mu, T)$ such that for every integer $l>1$ there exists a set $A=A(l) \in \mathscr{B}$ with $\mu(A)>0$ and $\mu\left(A \cap T^{n} A \cap\right.$ $\left.T^{2 n} A \cap T^{3 n} A \cap T^{4 n} A\right) \leq \frac{1}{2} \mu(A)^{l}$.

We will describe now one more "nilpotent connection" recently established in [BL5]. The main object of study in [BL5] is the class of generalized polynomials, that is, functions obtained from conventional polynomials of one or several variables by applying the operations of addition, multiplication, and that of taking the integer part. Various classes of generalized polynomials naturally appear in diverse mathematical contexts, ranging from symbolic dynamics and mathematical games to Weyl's theorem on equidistribution ${ }^{11}$ and recent work of Green and Tao [GreT] on arithmetic progressions in primes. ${ }^{12}$

Before formulating a general result from [BL5] which links generalized polynomials with nilsystems, let us briefly review a dynamical approach, due to Furstenberg, to the proof of Weyl's equidistribution theorem (see [F4], [F2]). Let $p(x)=$ $a_{0}+a_{1} x+a_{2} x^{2}+\cdots+a_{k} x^{k}=b_{0}+b_{1} x+b_{2}\left(\begin{array}{l}x \\ 2\end{array}\right)+\cdots+b_{k}\left(\begin{array}{l}x \\ k\end{array}\right) \in \mathbb{R}[x]$. Consider the following affine transformation, called a skew product, of the $k$-dimensional torus $\mathbb{T}^{k}=\mathbb{R}^{k} / \mathbb{Z}^{k}$ :

$$
\tau\left(y_{1}, t_{2}, \ldots, y_{k}\right)=\left(y_{1}+b_{k}, y_{2}+y_{1}+b_{k-1}, \ldots, y_{k}+y_{k-1}+b_{1}\right) .
$$

Let $y=\left(0, \ldots, 0, b_{0}\right) \in \mathbb{T}^{k}$. One can check by induction that $\left(\tau^{n} y\right)_{k}=\{p(n)\}$. If $a_{k}$ is irrational, the system ( $\mathbb{T}^{k}, \tau$ ) is uniquely ergodic (with the unique $\tau$-invariant measure being the Lebesgue measure on $\mathbb{T}^{k}$ ) which implies (the one-dimensional version of) Weyl's theorem. (For details, see [F4], Chapter 3, Section 3.)

One can also view the skew product transformation $\tau$ as a nilrotaion. Indeed, let $G$ be the group of upper triangular matrices $\left(\begin{array}{ccccc}1 & \alpha_{1,2} & \alpha_{1,3} & \ldots & \alpha_{1, k} \\ 0 & 1 & \alpha_{2,3} & \ldots & \alpha_{2, k} \\ \vdots & \ddots & \ddots & \ddots & \vdots \\ 0 & \cdots & 0 & 1 & \vdots \\ 0 & 0 & \cdots & 0 & a_{k, k+1}\end{array}\right)$, where $a_{i, j} \in \mathbb{Z}$ for

\footnotetext{
${ }^{11}$ Weyl's theorem (or rather the most quotable special case of it) says that if $p$ is a real polynomial with at least one coefficient other than the constant term irrational then the sequence $\{p(n)\}=p(n)-[p(n)], n \in \mathbb{N}$ is uniformly distributed in the unit interval

${ }^{12}$ See, for example, [Gre], p. 13
} 
$1 \leq i<j \leq k, a_{i, k+1} \in \mathbb{R}$ for $\left.1 \leq i<k\right\}$, and let $\Gamma$ be the subgroup of $G$ consisting of the matrices with integer entries. Then $G$ is a nilpotent (non-connected) Lie group with $X=G / \Gamma \cong \mathbb{T}^{k}$, and the system defined on $X$ by the nilrotation by the element $g=\left(\begin{array}{ccccc}1 & 0 & 0 & \ldots & b_{k} \\ 0 & 1 & 0 & \ldots & b_{k-1} \\ \vdots & \ddots & \ddots & \vdots & \vdots \\ 0 & \cdots & 0 & 1 & b_{1} \\ 0 & 0 & \cdots & 0 & 1\end{array}\right) \in G$ is isomorphic to the dynamical system on $\mathbb{T}^{k}$ defined by the skew product $\tau$.

The following result obtained in [BL5] says, roughly, that not only generalized polynomials of the special form $\{p(n)\}=p(n)-[p(n)]$, but any bounded generalized polynomial can be "read off" of a nilmanifold.

Theorem 4.2. For all $d \in \mathbb{N}$ and all bounded generalized polynomials $p: \mathbb{Z}^{d} \rightarrow \mathbb{R}$ there exists a compact nilmanifold $X$, an ergodic $\mathbb{Z}^{d}$ action $\left(T_{n}\right)_{n \in \mathbb{Z}^{d}}$ by nilrotations on $X$, a Riemann integrable function $f$ on $X$ and a point $x \in X$ such that for all $n \in \mathbb{Z}^{d}$ one has $p(n)=f\left(T_{n} x\right)$.

Here is one of the numerous corollaries of Theorem 4.2:

Theorem 4.3. Let $k \in \mathbb{N}$, let $U_{1}, \ldots, U_{k}$ be commuting unitary operators on a Hilbert space and let $p_{1}, \ldots, p_{k}$ be generalized polynomials $\mathbb{Z}^{d} \rightarrow \mathbb{Z}$. For any Folner sequence ${ }^{13}\left(\Phi_{N}\right)_{N=1}^{\infty}$ in $\mathbb{Z}^{d}$ the sequence

$$
\frac{1}{\left|\Phi_{N}\right|} \sum_{n \in \Phi_{N}} U_{1}^{p_{1}(n)} \ldots U_{k}^{p_{k}(n)}
$$

is convergent in the strong operator topology.

Theorem 4.3 leads to the following conjecture.

Conjecture 4.4. Theorem 4.3 remains true if the operators $U_{1}, \ldots, U_{k}$ appearing in its formulation generate a nilpotent group.

Assume now that the unitary operators $U_{1}, \ldots, U_{k}$ are induced by commuting measure preserving transformations $T_{1}, \ldots, T_{k}$ acting on a probability space $(X, \mathscr{B}, \mu)$. In this case it is natural to inquire under which conditions on the generalized integervalued polynomials $p_{i}$ one has

$$
\lim _{N-M \rightarrow \infty} \frac{1}{N-M} \sum_{n=M}^{N-1} \mu\left(A \cap T_{1}^{p_{1}(n)} T_{2}^{p_{2}(n)} \ldots T_{k}^{p_{k}(n)} A\right)>0
$$

for all $A \in \mathscr{B}$ with $\mu(A)>0$. In the case of conventional integer-valued polynomials a satisfactory sufficient condition for positivity of the above limit is that $p_{i}(0)=0$,

\footnotetext{
${ }^{13} \mathrm{~A}$ sequence $\left(\Phi_{N}\right)_{N=1}^{\infty}$ of finite subsets of a (countable) group $G$ is called (left) F $\phi$ lner if for all $g \in$ $G,\left|g \Phi_{N} \cap \Phi_{N}\right| /\left|\Phi_{N}\right| \rightarrow 1$ as $N \rightarrow \infty$. In $\mathbb{Z}^{d}$ a common choice of Følner sequence is a sequence of parallelepipeds $\Phi_{N}=\prod_{i=1}^{d}\left[a_{N, i}, b_{N, i}\right]$ with $b_{N, i}-a_{N, i} \rightarrow \infty$ as $N \rightarrow \infty$ for all $i=1, \ldots, d$.
} 
$i=1, \ldots, k$; the following conjecture extends this fact to generalized polynomials. Let us denote by $\mathcal{P}_{0}$ the set of generalized polynomials which can be constructed (with the help of addition, multiplication, and taking of integer part) from conventional polynomials with zero constant term.

Conjecture 4.5. Let $k \in \mathbb{N}$ and $p_{1}, \ldots, p_{k} \in \mathcal{P}_{0}$. Then for any commuting invertible measure preserving transformations $T_{1}, \ldots, T_{k}$ of a probability measure space

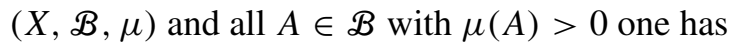

$$
\lim _{N-M \rightarrow \infty} \frac{1}{N-M} \sum_{n=M}^{N-1} \mu\left(A \cap T_{1}^{p_{1}(n)} T_{2}^{p_{2}(n)} \ldots T_{k}^{p_{k}(n)} A\right)>0 .
$$

Note that Conjecture 4.5 implies that $\left\{n: \mu\left(A \cap T_{1}^{p_{1}(n)} T_{2}^{p_{2}(n)} \ldots T_{k}^{p_{k}(n)} A\right)>0\right\}$ is a syndetic set. This, in turn, is a special case of the following conjecture, which extends the polynomial Szemerédi theorem (cf. Theorem 2.4 above) to generalized polynomials belonging to $\mathcal{P}_{0}$.

Conjecture 4.6. Let $(X, \mathscr{B}, \mu)$ be a probability measure space, let $k, r \in \mathbb{N}$, let $T_{1}, \ldots, T_{k}$ be commuting invertible measure preserving transformations of $X$ and let $p_{i, j} \in \mathcal{P}_{0}, i=1, \ldots, k, j=1, \ldots, r$. Then for all $A \in \mathscr{B}$ with $\mu(A)>0$, the set

$$
\left\{n \in \mathbb{Z}: \mu\left(A \cap T_{1}^{p_{1,1}(n)} \ldots T_{k}^{p_{k, 1}^{(n)}} A \cap \cdots \cap T_{1}^{p_{1, r}(n)} \ldots T_{k}^{p_{k, r}^{(n)}} A\right)>0\right\}
$$

is syndetic in $\mathbb{Z}$.

\section{Amenable groups and ergodic Ramsey theory}

It was John von Neumann who, in his study of the Hausdorff-Banach-Tarski paradox, introduced a class of group which nowadays are called amenable and which are widely recognized as providing the natural context for ergodic theory. In particular, many classical notions and results pertaining to 1-parameter group actions extend naturally to amenable groups. (See for example [OW] and [Li]). As we will see in this section, countable amenable groups also form a natural framework for Furstenberg's correspondence principle and hence for ergodic Ramsey theory.

Definition 5.1. A semigroup $G$ is amenable if there exists an invariant mean on the space $B(G)$ of real-valued bounded functions on $G$, that is, a positive linear function $L: B(G) \rightarrow \mathbb{R}$ satisfying

(i) $L\left(\mathbf{1}_{G}\right)=1$.

(ii) $L\left(f_{g}\right)=L\left({ }_{g} f\right)=L(f)$ for all $f \in B(G)$ and $g \in G$, where $f_{g}(t):=f(t g)$ and $g f(t):=f(g t)$. 
The existence of an invariant mean is only one item from a long list of equivalent properties, such as the characterization of amenability given in the next theorem, some of which are far from being obvious and, moreover, are valid for groups (or special classes of semigroups) only. (See, for example, [W], Theorem 10.11.) The following theorem was established by Følner in [Fø]. (See also [N] for a simplified proof.)

Theorem 5.2. A countable group $G$ is amenable if and only if it has a left Fфlner sequence, namely a sequence of finite sets $\Phi_{n} \subset G, n \in \mathbb{N}$, with $\left|\Phi_{n}\right| \rightarrow \infty$ and such that for all $g \in G, \frac{\left|g \Phi_{n} \cap \Phi_{n}\right|}{\left|\Phi_{n}\right|} \rightarrow 1$ as $n \rightarrow \infty$.

While there seems to be no general method of constructing a Følner sequence in an amenable group defined, say, by generators and relations, in many concrete, especially abelian situations, one has no problem finding a Følner sequence. For example, the parallelepipeds mentioned in footnote 13 and the sets $F_{n} \subset F_{\infty}$ defined in section 1 form natural Følner sequences in $\mathbb{Z}^{d}$ and in $F_{\infty}$, respectively.

Before moving to discuss the Ramsey-theoretical aspects of amenable groups we want to mention that while the class of amenable groups is quite rich (in particular it contains all solvable and locally finite groups), it does not contain such classical groups as $\operatorname{SL}(n, \mathbb{Z})$ for $n \geq 2$.

Given a countable amenable group $G$ and a left Følner sequence $\left(\Phi_{n}\right)_{n \in \mathbb{N}}$, one can define the upper density of a set $E \subset G$ with respect to $\left(\Phi_{n}\right)_{n \in \mathbb{N}}$ by $\bar{d}_{\left(\Phi_{n}\right)}(E)=$ $\lim \sup _{n \rightarrow \infty} \frac{\left|E \cap \Phi_{n}\right|}{\left|\Phi_{n}\right|}$. Note that it immediately follows from the definition of a left Følner sequence that for all $g \in G$ and $E \subset G$ one has $\bar{d}_{\left(\Phi_{n}\right)}(g E)=\bar{d}_{\left(\Phi_{n}\right)}(E)$. By analogy with some known results about sets of positive density in abelian or nilpotent groups, one can expect that large sets in $G$, i.e. sets having positive upper density with respect to some Følner sequence, will contain some nontrivial configurations. The known results support this point of view and lead to a natural conjecture which will be formulated at the end of this section.

We formulate now a version of Furstenberg's correspondence principle for countable amenable groups.

Theorem 5.3 (See [B2], Theorem 6.4.17). Let $G$ be a countable amenable group and assume that $E \subset G$ has positive upper density with respect to some left Folner sequence $\left(\Phi_{n}\right)_{n \in \mathbb{N}}: \bar{d}_{\left(\Phi_{n}\right)}(E)>0$. Then there exists a probabilitymeasure preserving system $\left(X, \mathscr{B}, \mu,\left(T_{g}\right)_{g \in G}\right)$ and a set $A \in \mathscr{B}$ with $\mu(A)=\bar{d}_{\left(\Phi_{n}\right)}(E)$ such that for all $k \in \mathbb{N}$ and $g_{1}, \ldots, g_{k} \in G$ one has

$$
\bar{d}_{\left(\Phi_{n}\right)}\left(E \cap g_{1} E \cap \cdots \cap g_{k} E\right) \geq \mu\left(A \cap T_{g_{1}} A \cap \cdots \cap T_{g_{k}} A\right) .
$$

Remark. One can extend Theorem 5.3 to general countable amenable semigroups if instead of using Følner sequences (which cannot always be found in amenable semigroups) one defines a set $E \subset G$ to be large if for some left-invariant mean $L$ on $B(G)$ one has $L\left(1_{E}\right)>0$. (See [BM1], Theorem 2.1.) 
As an illustration of the usefulness of amenable considerations, let us consider the (abelian and cancellative) semigroup $(\mathbb{N}, \cdot)$. Let

$$
S_{n}=\left\{p_{1}^{i_{1}} p_{2}^{i_{2}} \cdots p_{n}^{i_{n}}, 0 \leq i_{j} \leq n, 1 \leq j \leq n\right\},
$$

where $p_{i}, i=1,2, \ldots$, are primes in arbitrary order. It is not hard to show that for any sequence of positive integers $\left(a_{n}\right)_{n \in \mathbb{N}}$, the sets $a_{n} S_{n}, n \in \mathbb{N}$ form a Følner sequence in $(\mathbb{N}, \cdot)$.

Definition 5.4. A set $E \subset \mathbb{N}$ is called multiplicatively large if for some Følner sequence $\left(\Phi_{n}\right)_{n \in \mathbb{N}}$ in $(\mathbb{N}, \cdot)$ one has $\bar{d}_{\left(\Phi_{n}\right)}(E)>0$.

Notice that the notions of additive and multiplicative largeness which are defined via Følner sets in, respectively, $(\mathbb{N},+)$ and $(\mathbb{N}, \cdot)$ are different. For example the set $O$ of odd natural numbers has additive density $\frac{1}{2}$ with respect to every Følner sequence in $(\mathbb{N},+)$, while $O$ has zero density with respect to every Følner sequence in $(\mathbb{N}, \cdot)$. In the other direction, consider for example a Følner sequence $\left(a_{n} S_{n}\right)_{n \in \mathbb{N}}$ in $(\mathbb{N}, \cdot)$, where the $S_{n}$ are defined above and the $a_{n}$ satisfy $a_{n}>\left|S_{n}\right|$. Then the set $E=\bigcup_{n=1}^{\infty} a_{n} S_{n}$ has zero additive density with respect to every Følner sequence in $(\mathbb{N},+)$, while $E$ has multiplicative density 1 with respect to the Følner sequence $\left(a_{n} S_{n}\right)_{n \in \mathbb{N}}$.

As may be expected by mere analogy with additively large sets, multiplicatively large sets always contain (many) geometric progressions. (This can be derived, for example, with the help of the IP Szemerédi theorem, Theorem 1.13 above). It turns out, however, that multiplicatively large sets also contain some other, somewhat unexpected geoarithmetic configurations.

Theorem 5.5 (See [B5], Theorems 3.11 and 3.15). Let $E \subset \mathbb{N}$ be a multiplicatively large set. For all $k \in \mathbb{N}$, there exist $a, b, c, d, e, q \in \mathbb{N}$ such that $\left\{q^{i}(a+i d): 0 \leq\right.$ $i, j \leq k\} \subset E$ and $\left\{b(c+i e)^{j}: 0 \leq i, j \leq k\right\} \subset E$.

We conclude this section (and this survey) by addressing the question about possible amenable extensions of the multiple recurrence results. While it is not clear how even to formulate an amenable generalization of the one-dimensional Szemerédi theorem, it is not too hard to guess what should be an amenable version of the multidimensional Szemerédi theorem!

Let $G$ be a group and $k \in \mathbb{N}$. Let us call a $(k+1)$-element set in the cartesian product $G^{k}$ a simplex if it is of the form

$$
\left\{\left(a_{1}, a_{2}, \ldots, a_{k}\right),\left(g a_{1}, a_{2}, \ldots, a_{k}\right),\left(g a_{1}, g a_{2}, \ldots, a_{k}\right), \ldots,\left(g a_{1}, g a_{2}, \ldots, g a_{k}\right)\right\}
$$

for some $a_{1}, \ldots, a_{k}, g \in G$, and denote it by $S\left(a_{1}, \ldots, a_{k} ; g\right)$. The following conjecture is known for $k=2$. (See [BMZ], Theorem 6.1.)

Conjecture 5.6. Let $k \in \mathbb{N}$ and suppose that $G$ is a countable amenable group. Assume that a set $E \subset G^{k}$ has positive upper density with respect to some Følner 
sequence in $G^{k}$. Then the set

$\left\{g \in G:\right.$ there exist $\left(a_{1}, \ldots, a_{k}\right) \in G^{k}$ such that $\left.S\left(a_{1}, \ldots, a_{k} ; g\right) \subset E\right\}$

is syndetic in $G$.

\section{References}

[B1] Bergelson, V., Ergodic Ramsey theory - an update. In Ergodic Theory of $\mathbb{Z}^{d}$-actions (Warwick, 1993-1994), London Math. Soc. Lecture Note Ser. 228, Cambridge University Press, Cambridge 1996, 273-296.

[B2] Bergelson, V., Ergodic theory and diophantine problems. In Topics in Symbolic Dynamics and Applications (Temuco 1997), London Math. Soc. Lecture Note Ser. 277, Cambridge University Press, Cambridge 2000, 167-205.

[B3] Bergelson, V., Minimal idempotents and ergodic Ramsey theory. In Topics in Dynamics and Ergodic Theory, London Math. Soc. Lecture Note Ser. 279, Cambridge University Press, Cambridge 2000, 167-205.

[B4] Bergelson, V., Combinatorial and diophantine applications of ergodic theory. Appendix A by A. Leibman and Appendix B by A. Quas and M. Wierdl. In Handbook of Dynamical Systems (ed. by B. Hasselblatt and A. Katok), Vol. 1B, Elsevier, Amsterdam 2006, 745-841.

[B5] Bergelson, V., Multiplicatively large sets and ergodic Ramsey theory. Israel J. Math. 148 (2005), 23-40.

[BBHi] Bergelson, V., Blass, A., Hindman, N., Partition theorems for spaces of variable words. Proc. London Math. Soc. (3) 68 (3) (1994), 449-476.

[BFM] Bergelson, V., Furstenberg, H., McCutcheon, R., IP-sets and polynomial recurrence. Ergodic Theory Dynam. Systems 16 (5) (1996), 963-974.

[BHKR] Bergelson, V., Host, B., Kra, B., Multiple recurrence and nilsequences. (With an appendix by I. Ruzsa.) Invent. Math. 160 (2) (2005), 261-303.

[BL1] Bergelson, V., Leibman, A., Polynomial extensions of Van der Waerden's and Szemerédi's theorems. J. Amer. Math. Soc. 9 (1996), 725-753.

[BL2] Bergelson, V., Leibman, A., Set-polynomials and polynomial extension of the HalesJewett theorem. Ann. of Math. (2) 150 (1) (1999), 33-75.

[BL3] Bergelson, V., Leibman A., Topological multiple recurrence for polynomial configurations in nilpotent groups. Adv. Math. 175 (2) (2003), 271-296.

[BL4] Bergelson, V., Leibman, A., Failure of the Roth theorem for solvable groups of exponential growth. Ergodic Theory Dynam. Systems. 24 (2004), 45-53.

[BL5] Bergelson, V., Leibman, A., Distribution of values of bounded generalized polynomials. Submitted.

[BLM] Bergelson, V., Leibman A., McCutcheon, R., Polynomial Szemerédi theorems for countable modules over integral domains and finite fields. J. Anal. Math. 95 (2005) 243-296. 
[BM1] Bergelson, V., McCutcheon, R., Recurrence for semigroup actions and a noncommutative Schur Theorem. In Topological Dynamics and Applications (Minneapolis, MN, 1995), Contemp. Math. 215, Amer. Math. Soc., Providence, RI, 1998, 205-222.

[BM2] Bergelson, V., McCutcheon, R., An ergodic IP polynomial Szemerédi theorem. Mem. Amer. Math. Soc. 146 (695) (2000).

[BMZ] Bergelson, V., McCutcheon, R., Zhang, Q., A Roth theorem for amenable groups. Amer. J. Math. 119 (6) 1997, 1173-1211.

[BPT] Blaszczyk, A., Plewik, S., Turek, S., Topological multidimensional van der Waerden theorem. Comment. Math. Univ. Carolin. 30 (4) (1989), 783-787.

[C] Carlson, T., Some unifying principles in Ramsey theory. Discrete Math. 68 (1988), 117-169.

[CN] Comfort, W., Negrepontis, S., The Theory of Ultrafilters. Grundlehren Math. Wiss. 211, Springer-Verlag, Berlin 1974.

[E] Ellis, R., A semigroup associated with a transformation group. Trans. Amer. Math. Soc. 94 (1960), 272-281.

[Fø] Følner, E., On groups with full Banach mean values. Mat. Scand. 3 (1955), 243-354.

[F1] Furstenberg, H., Stationary processes and prediction theory. Ann. of Math. Stud. 44, Princeton University Press, Princeton, N.J., 1960

[F2] Furstenberg, H., The structure of distal flows. Amer. J. Math. 85 (1963), 477-515.

[F3] Furstenberg, H., Ergodic behavior of diagonal measures and a theorem of Szemerédi on arithmetic progressions. J. Anal. Math. 31 (1977), 204-256.

[F4] Furstenberg, H., Recurrence in Ergodic Theory and Combinatorial Number Theory. Princeton University Press, Princeton, N.J., 1981.

[FK1] Furstenberg, H., Katznelson, Y., An ergodic Szemerédi theorem for commuting transformations. J. Anal. Math. 34 (1978), 275-291.

[FK2] Furstenberg, H., Katznelson, Y., An ergodic Szemerédi theorem for IP-systems and combinatorial theory. J. Anal. Math. 45 (1985), 117-168.

[FK3] Furstenberg, H., Katznelson, Y., A density version of the Hales-Jewett theorem. J. Anal. Math. 57 (1991), 64-119.

[FW] Furstenberg, H., Weiss, B., Topological dynamics and combinatorial number theory. J. Anal. Math. 34 (1978), 61-85.

[GraRS] Graham, R., Rothschild, B., Spencer, J., Ramsey Theory. Wiley, New York 1980.

[Gre] Green, B. Long arithmetic progressions of primes. Available at http://arxiv.org/ abs/math.NT/0508063.

[GreT] Green, B., Tao, T., The primes contain arbitrarily long arithmetic progressions. Ann. of Math., to appear.

[Gri] Grigorchuk, R. I., Degrees of growth of finitely generated groups and the theory of invariant means. Izv. Akad. Nauk SSSR, Ser 1 Mat. 48 (1984) (5) 939-985; English transl. Math. USSR-Izv. 25 (2) (1985), 259-300.

[HaJ] Hales, A. W., Jewett, R. I. Regularity and positional games. Trans. Amer. Math. Soc. 106 (1963), 222-229.

[Hi] Hindman, N., Finite sums from sequences within cells of a partition of $\mathbb{N}$. J. Combin. Theory (Ser. A) 17 (1974), 1-11. 
[HiS] Hindman, N., Strauss, D., Algebra in the Stone-Čech compactification. Theory and Applications. De Gruyter Exp. Math. 27, Walter de Gruyter, Berlin 1998.

[HK1] Host, B., Kra, B., Nonconventional ergodic averages and nilmanifolds. Ann. of Math. 161 (2005) (1), 397-488.

[HK2] Host, B., Kra, B., Convergence of polynomial ergodic averages. Israel J. of Math. 148 (2005), 267-276.

[KM] Kamae, T., Mendès-France, M., Van der Corput's difference theorem. Israel J. Math. 31 (3-4) (1978), 335-342.

[L1] Leibman, A., Multiple recurrence theorem for measure preserving actions of a nilpotent group. Geom. Funct. Anal. 8 (1998), 853-931.

[L2] Leibman, A., Pointwise convergence of ergodic averages for polynomial sequences of transformations on a nilmanifold. Ergodic Theory Dynam. Systems 25 (2005), 201-213.

[L3] Leibman, A., Pointwise convergence of ergodic averages for polynomial actions of $\mathbb{Z}^{d}$ by translations on a nilmanifold. Ergodic Theory Dynam. Systems 25 (2005), 215-225.

[L4] Leibman, A. Convergence of multiple ergodic averages along polynomials of several variables. Israel J. Math. 146 (2005), 303-315.

[L5] Leibman, A., Orbits on nilmanifolds under the actions of polynomial sequences of translations. Preprint; http://www.math.ohio-state.edu/ leibman/preprints/.

[Li] Lindenstrauss, E., Pointwise theorems for amenable groups. Invent. Math. 146 (2001), 259-295.

[M] Milliken, K., Ramsey's theorem with sums or unions. J. Combin. Theory (Ser. A) 18 (1975), 276-290.

[N] Namioka, I., Følner's condition for amenable semi-groups. Math. Scand. 15 (1964), 18-28.

[O] Ornstein, Donald S., Bernoulli shifts with the same entropy are isomorphic. Adv. Math. 4, 337-352.

[OW] Ornstein, D., Weiss, B., Entropy and isomorphism theorems for actions of amenable groups. J. Anal. Math. 48 (1987), 1-141.

[R] Rado, R., Note on combinatorial analysis. Proc. London Math. Soc. 48 (1993), 122-160.

[Ro] Rosenblatt, J., Invariant measures and growth conditions. Trans. Amer. Math. Soc. 193 (1974), 33-53.

[Sa] Sàrközy, On difference sets of integers III. Acta Math. Acad. Sci. Hungar. 31 (1978) 125-149.

[Sz] Szemerédi, E., On sets of integers containing no $k$ elements in arithmetic progression. Acta Arith. 27 (1975), 199-245.

[T] Taylor, A., A canonical partition relation for finite subsets of $\omega$. J. Combin. Theory (Ser A.) 17 (1974), 1-11.

[W] Wagon, S., The Banach-Tarski Paradox. Cambridge University Press, Cambridge 1985.

[Z] Ziegler, T., Universal characteristic factors and Furstenberg averages. J. Amer. Math. Soc., to appear.

Department of Mathematics, Ohio State University, Columbus, OH 43210, U.S.A.

E-mail: vitaly@math.osu.edu 